\title{
CHARGE WAVES IN DOUBLE DOPED PHOTOCHROMIC LITHIUM NIOBATE CRYSTALS
}

\author{
RUBEN HOVSEPYAN, ARMEN POGHOSYAN*, EDUARD VARDANYAN \\ Institute for Physical Research, Armenian National Academy of Sciences \\ Ashtarak-2, 0203, Republic of Armenia \\ *arp@ipr.sci.am
}

\begin{abstract}
We are presenting the experimental studies of time-space characteristics of photochromic effect in lithium niobate crystals with double doping. The photoinduced autowaves of absorption coefficient changes under the light illumination of limited part of crystal were found. An analytical model is proposed for the explanation of appearance and spreading of charge waves.
\end{abstract}

Keywords: Lithium niobate; photochromic effect; charge waves.

\section{Introduction}

Spatial separation of the charges takes place under the illumination of some limited region of double doped ferroelectric lithium niobate crystals $\left(\mathrm{LiNbO}_{3}: \mathrm{Fe}: \mathrm{Cu}\right.$, $\left.\mathrm{LiNbO}_{3}: \mathrm{Fe}: \mathrm{Mn}\right)$. That is induced, mainly, because of photovoltaic and diffuse mechanisms and formation of electric fields, which lead to photorefractive effect - to the change of refraction index ${ }^{1}$. Beside the redistribution of electrons among the different types of impurity centers takes place leading to photochromic effect - to the change of absorption coefficient ${ }^{2}$. In Refs. 3 and 4 it was shown, that the photochromic effect has the light intensity threshold and the model for this effect based on the charge redistribution among different types of dopants was proposed.

In this work the experimental studies of time-space characteristics of the photochromic effect are presented. It is found that changes of the absorption coefficient have explicitly outlined oscillating character. The photoinduced autowaves of alterations in absorption coefficient under the photoactive light illumination of some limited part of crystal are studied. The attempt is made to clear up the dynamic of the system along with changes of external factors and parameters of crystal. An analytical model is proposed for the explanation of appearance and spreading of autowaves in the wide band of light intensities.

\section{Experimental Results}

The experiments on the study of space-time characteristics of photochromic effect were conducted as following. The light beam from argon laser with wavelength $488 \mathrm{~nm}$ and 
power $0-1000 \mathrm{~mW}$ passing through the system of attenuators and beam formatter interacted with crystal sample. Space distribution of absorption coefficient was measured by TV system of "BUEHLER" firm. Autowaves by its time parameter were studied with the help of photodetector directed to the small part of illuminated region. As samples we used the crystal plates of $\mathrm{LiNbO}_{3}$ doped by Fe:Cu and Fe:Mn impurities. The polar axis of crystal was parallel to the crystal surface, on which the laser light falls. The polarization of the light was parallel to the polar axe. The used samples had sizes $10 * 10 * 2 \mathrm{~mm}^{3}$; the illuminated region had $2 \mathrm{~mm}$ diameter. To except discharges of induced electric fields the crystal surface was covered by liquid siliciumorganic compound with high discharge tension and small dielectric losses. Liquid insulator was covered by the defending glass. The signal from photodetector was digitized and inputted to computer.
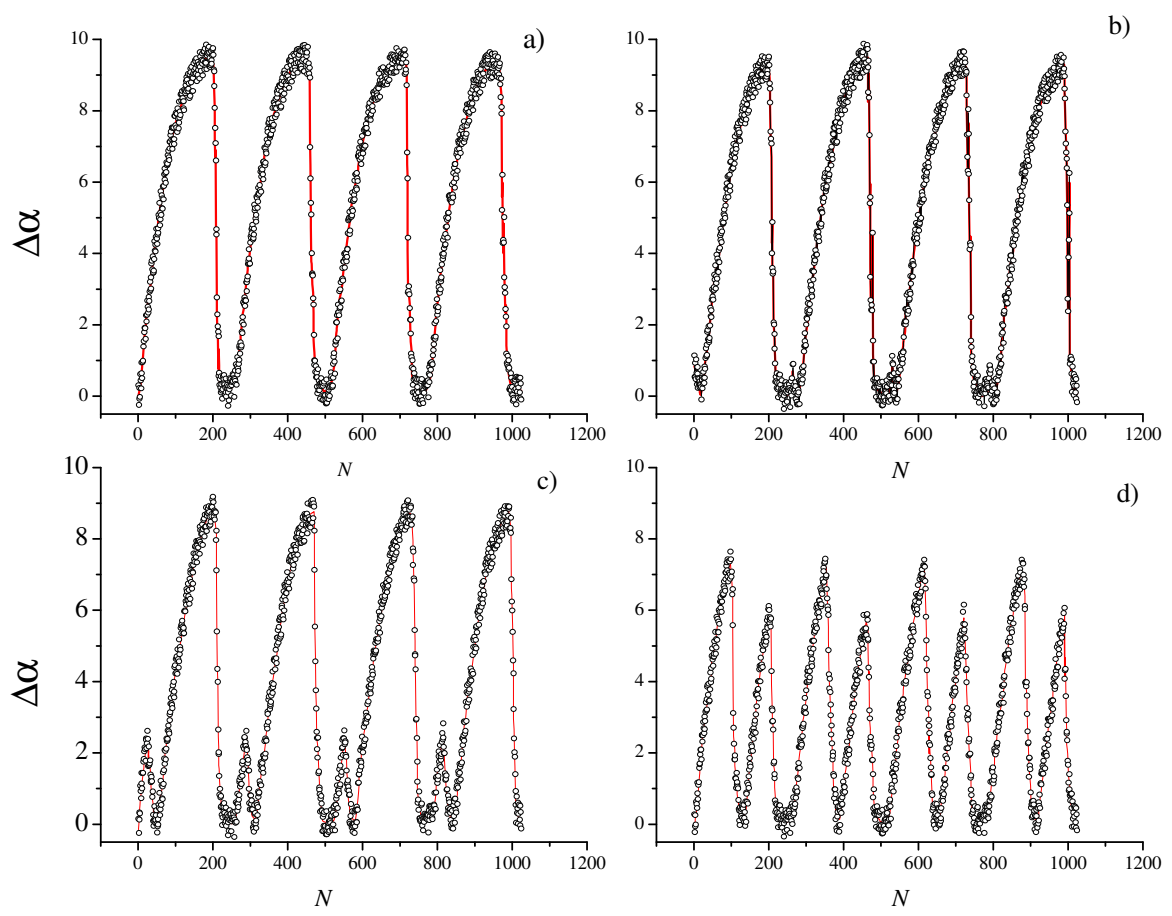

Fig. 1. Dependences of oscillating components of crystal absorption coefficient changes on time $t=\mathrm{T}_{\mathrm{c}} \times \mathrm{N}$ $\left(\mathrm{T}_{\mathrm{c}}=2.5 \mathrm{sec}\right)$ at different light intensities: $\mathrm{I}_{1}<\mathrm{I}_{2}<\mathrm{I}_{3}<\mathrm{I}_{4}\left(\mathrm{a}-\mathrm{I}_{1}, \mathrm{~b}-\mathrm{I}_{2}, \mathrm{c}-\mathrm{I}_{3}, \mathrm{~d}-\mathrm{I}_{4}\right)$.

The space-time autowaves moving in the direction of polar axes were observed. Because of technical difficulties in the experiment we measured mainly time waves, i.e. the magnitude of signal was measured in one point of space. The signal discretization was carried out in constant time periods $T$. The discretization frequency $(F=1 / T)$ was chosen as of 4-8 times more than the maximal frequency of studied signal spectrum. To except statistical errors and to increase accuracy of calculation the discretization was 
carried out for some vibration period. The measured in the experiment signal included two main components, caused by different interaction mechanisms. First component was caused by decreasing of the intensity of the light passing through the crystal due to the increasing of absorption coefficient $(\Delta \alpha)$ induced by laser. This component was approximated by attenuating exponent via method of minimal squares and was subtracted from the obtained signal. As a result the second component of signal was provided only by oscillating components of photochromic effect.

In the Fig.1 the time dependencies of oscillating component of the absorption coefficient change for different intensities of exciting radiation are presented. Building of phase trajectories and spectral analysis were carried out by digital method. Preliminarily the obtained dependencies were subject to digital filtration ${ }^{5}$ for removing of high frequency noises and increasing of accuracy of phase trajectories. As a digital filter we used nonrecursive filter with approximating polynom of fourth order. The transparency band of used filter was $0.2 * \mathrm{~F}$ with transparency maximum at zero frequency; the Nyquist frequency $\mathrm{g}$ is $0.9 * \mathrm{~F}$. Thus the high frequency noises which are present in the signal and carry no information about the physical processes taking place in the crystal were cut.

a)

b)
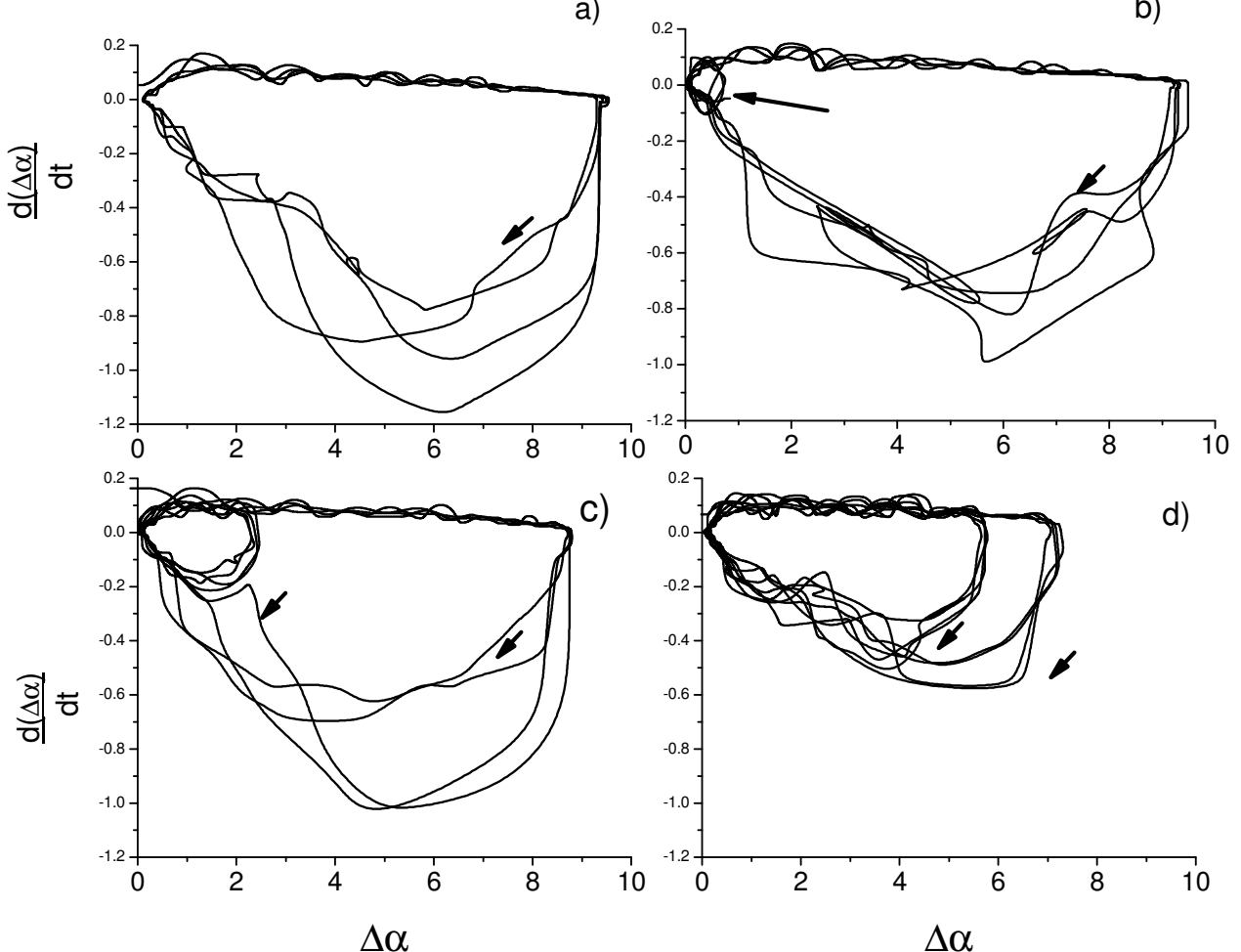

Fig. 2. Phase portraits of the system for oscillating component of absorption coefficient at different light intensities: $\mathrm{I}_{1}<\mathrm{I}_{2}<\mathrm{I}_{3}<\mathrm{I}_{4}\left(\mathrm{a}-\mathrm{I}_{1}, \mathrm{~b}-\mathrm{I}_{2}, \mathrm{c}-\mathrm{I}_{3}, \mathrm{~d}-\mathrm{I}_{4}\right)$. 
Fig.2 shows the phase portraits of the system for different light intensities. These dependences are obtained from the experimental data presented in Fig. 1. Calculation of first derivative for building of phase trajectories was carried out with use of differentiating nonrecursive filter (with maximum at frequency $0.15^{*} \mathrm{~F}$ ). Spectral analysis was carried out via discrete Fourier-transformation.

In Fig. 3 the amplitudes of the harmonics of the Fourier spectrum calculated according to Hamming algorithm are presented.
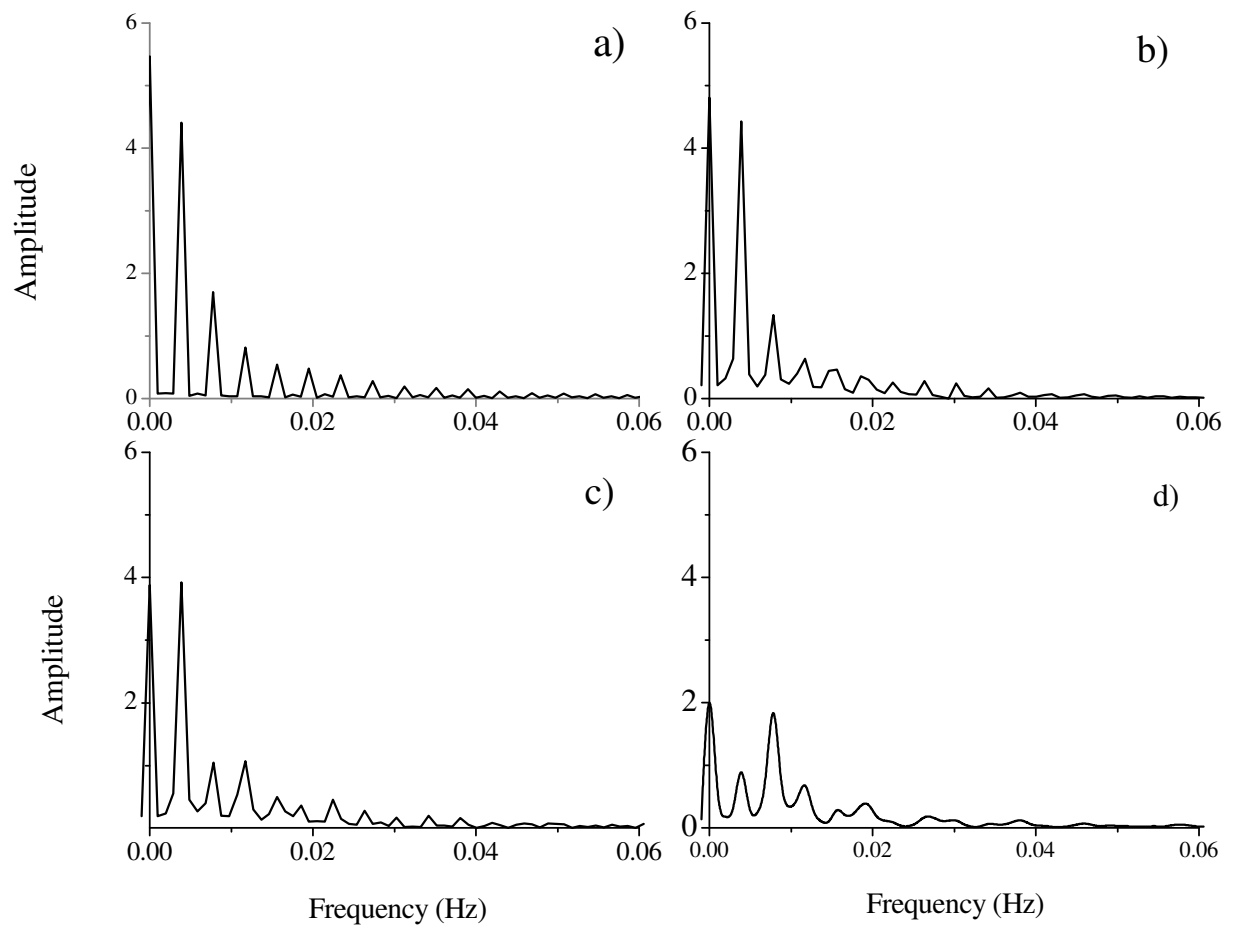

Fig. 3. Fourier spectrum of the oscillating component of absorption coefficient at different light intensities: $\mathrm{I}_{1}<\mathrm{I}_{2}<\mathrm{I}_{3}<\mathrm{I}_{4}\left(\mathrm{a}-\mathrm{I}_{1}, \mathrm{~b}-\mathrm{I}_{2}, \mathrm{c}-\mathrm{I}_{3}, \mathrm{~d}-\mathrm{I}_{4}\right)$.

\section{Discussion}

In our experiments the bifurcations were observed on the increasing of exciting laser intensity. As it is shown in Ref. 2, at small light intensities the change of transmission coefficient doesn't take place. When the light intensity is increased the threshold change of absorption coefficient (or the shift of stable focus from its initial point to other one) takes place. At further increasing of light intensity (at intensities $0.2 \mathrm{~W} / \mathrm{cm}^{2}$ ) the waves moving in the direction of crystal polar axes are appeared. Below this intensity there are no oscillations. In other words, it is observed Hopf bifurcation ${ }^{6}$, i.e. transition of dynamic system from stable focus on the phase plane to the close phase trajectory with appearance of limiting cycle. 
Further increase of light intensity leads to the increase of phase trajectory area and its approaching to the unstable limiting cycle. Further the bifurcation takes place with splitting of phase trajectory into two ones with less area (Fig. 3). In contrast to known scenarios of transition to the chaos with doubling of the period, in the observed bifurcation the doubling of the frequency takes place. In our experiments the power of exciting laser was sufficient for observation of series of bifurcations (up to 6 bifurcations of frequency doubling); they observed in the intensity range $0.25-30 \mathrm{~W} / \mathrm{cm}^{2}$.

The scenario of transition to chaos through the unlimited chain of period doubling assumes that the interval of change of impact parameter, in which the limiting cycle with period $2^{N}$ exists, gets narrow with growth of $N$ according to Feigenbaum law ${ }^{7}: I_{n} / I_{n+1}=\delta$, where $\delta=4.6692$ is the universal Feigenbaum constant. In our studies, in contrast to other known mechanisms of period doubling, the doubling of frequency takes place. The interval of changes of excitation intensity between two bifurcations gets narrow with intensity growth as a geometrical progression, i.e. along with Feigenbaum law. The value of Feigenbaum constant, experimentally obtained, is 5-6.

For the qualitative description of observed autowaves let consider the mechanism of photochromic effect formation. In the Refs 2 and 4 the model of photochromic effect based on the charge transfer between $\mathrm{Fe}$ and $\mathrm{Cu}$ impurity centers is proposed; the charge transfer takes place due to photoelectron thanks to impurity - conducting band - impurity transitions. The space shift of the photoelectrons in the conducting band is caused by photovoltaic effect. Within the time of photoelectron impulse izotropization the space shift of photoelectron wave function takes place $^{8}$. As the $\mathrm{Cu}$ level is situated higher than Fe level, the change of absorption coefficient (at $l=488 \mathrm{~nm}$ ) is caused by the occupation of $\mathrm{Cu}$ level, and the rate of occupation of this level is defined by the concentration of photoelectrons in the conducting band.

Let's assume, that intrinsic light forms an illuminated region at $0<\mathrm{x}<\mathrm{L}$, and the size of crystal is much more than this region. In this area with the velocity $\beta \alpha I$ ( $\beta$ - quantum yield, $\alpha$ - absorption coefficient, I - light intensity) the photoelectrons with the concentration $\mathrm{n}(\mathrm{x}, \mathrm{t})$ and the mean impulse, directed along crystal polar axis $(\mathrm{Cll})$, are generated. The continuity equation for the photoelectrons in the conducting band has a form:

$$
\frac{\partial n}{\partial t}=-\frac{n}{\tau}-\frac{1}{e} \frac{\partial J(x, t)}{\partial x}+I \alpha \beta,
$$

where $\tau$ - relaxation time for photoelectrons. In this equation we neglect the Fe level depletion since it is situated sufficiently close to the crystal valence band $(1.1 \mathrm{eV})^{9}$. The current of charges separation is caused by three components: drift current, diffuse current and specific for ferroelectrics photovoltaic one ${ }^{8}$ :

$$
J(x, t)=\sigma E-D e \frac{\partial n}{\partial x}+I \alpha k
$$


where $\sigma$ is crystal conductivity, E - electric field formed due to charges separation, $\mathrm{k}$ - the Glass constant, D - diffusion coefficient. Electrostatic field E according to Poison equation may be written as following:

$$
\frac{d E}{d x}=\frac{4 \pi}{\varepsilon} e\left(n(x, t)-n_{0}\right)
$$

where $\varepsilon$ - dielectric constant, $\mathrm{n}_{\mathrm{o}}$ - holes concentration on Fe level after photoionization. Substituting equation (2) into (1) and taking into account equation (3) we obtain:

$$
\frac{\partial n}{\partial t}=-\frac{n}{\tau}+\frac{4 \pi \sigma}{\varepsilon}\left(n-n_{0}\right)+D \frac{\partial^{2} n}{\partial x^{2}}+I \alpha .
$$

The obtained equation can be solved analytically. We shall seek the solution in the form of running impulse or front. Taking the velocity of front's motion as a constant and positive one let's introduce the running coordinate system $\xi=\mathrm{x}-\mathrm{vt}$. Then equation (4) may be rewritten as an ordinary differential equation:

$$
D n^{\prime \prime}-v n^{\prime}+\left(\frac{1}{\tau}-\frac{4 \pi}{\varepsilon} \sigma\right) n+I \alpha=0,
$$

This equation can be solved by operator method, if one has initial and boundary conditions for $n$. Let's denote $n(x, 0)=n_{1}, n(x, \infty)=n_{2}, n_{1}>n_{2}$. Hence $n^{\prime}(x, 0)=0$ (at $\left.\xi=0\right)$. In other words, we are looking for a solution in a form of running step from the unstable concentration $\mathrm{n}_{1}$ to $\mathrm{n}_{2}$. If the all coefficients are assumed as real ones, then the reflection in Laplace-space will look as following:

$$
D\left(S^{2} N-S n_{1}\right)+\left(\frac{4 \pi}{\varepsilon}+v\right) \cdot\left[S N-S n_{1}\right]+\frac{1}{\tau} N-\frac{I \alpha}{S}=0
$$

where $\mathrm{S}$ - parameter $\xi$, and $\mathrm{N}$ is the image of function $\mathrm{n}$ in the space of reflection's operators. After reverse transformation we can obtain analytical solution. But the special points of equation may be obtained without analytical solution. Equation (6) has special points depending on the sign of discriminant:

$$
\Delta=\left(\frac{4 \pi}{\varepsilon} e+v\right)^{2}-\frac{D}{\tau},
$$

If the value $\Delta$ is more than zero, then the differential equation hasn't vibrational solution; it is damped and aperiodic one. In this case the appearing small noises are falling down; the waves moving in the $\mathrm{C}$ direction don't occur. If the discriminant is negative, we obtain the solution in a complex plane; and the equation has strictly vibrational solution. In this case the occurring small noises are amplified; and the vibrational regime is reached. In case of $\Delta=0$ it is possible to calculate the minimal stable velocity of wave motion after bifurcation. 
Thus, the presented model based on the continuity equation for photoelectrons and the Poison equation explains the transition of the system from stationary mode to vibrational one, i.e. Hopf bifurcation, and is sufficiently well correlated with obtained experimental results.

\section{Acknowledgments}

This work was made possible in part by the research grants EN-2528 and EN-2951 from the Armenian National Science and Education Fund (ANSEF) based in New York, USA.

\section{References}

1. F. S. Chen, J. Appl. Phys. 40, 3389 (1969).

2. E. S. Vardanyan, R. K. Hovsepyan and A. R. Poghosyan, Sov. phys. Kristallografia 35, 900 (1990).

3. A. S. Bagdassarian, R. K. Hovsepyan and A. R. Poghosyan, Journal of Optoelectronics and Advanced Materials 5, 875 (2003).

4. G. T. Avanesyan, E. S. Vardanyan, R. S. Mikaelyan, R. K. Hovsepyan and A. R. Poghosyan, Phys. Stat. Sol.(a) 126, 245 (1991).

5. R. V. Hemming, Digital filters ("Nedra", Moscow, 1987).

6. J. Marry, Nonlinear differential equations in biology ("Mir", Moscow, 1983).

7. M. J. Feigenbaum, Los Alamos Science 1, 4 (1980).

8. V. M. Fridkin and B. N. Popov, Sov. Phys., Uspechi 126, 657 (1978).

9. I. S. Amadullin, V. A. Golenishev-Kutuzov and S. P. Mirinov, Sov. Phys. Sol. State 32, 1854 (1990). 